\title{
Near-Complete Genome Assembly of Alternaria brassicae-A Necrotrophic Pathogen of Brassica Crops
}

\author{
Sivasubramanian Rajarammohan, ${ }^{1}$ Deepak Pental, ${ }^{2}$ and Jagreet Kaur ${ }^{1, \dagger}$ \\ ${ }^{1}$ Department of Genetics, University of Delhi - South Campus, Delhi, India \\ ${ }^{2}$ Centre for Genetic Manipulation of Crop Plants, University of Delhi - South Campus, Delhi, India
}

\begin{abstract}
Alternaria brassicae, a necrotrophic fungal pathogen, causes Alternaria blight, an important disease of brassica crops. Although many Alternaria spp. have been sequenced, no genome information is available for $A$. brassicae, a monotypic lineage within the Alternaria genus. A highly contiguous genome assembly of $A$. brassicae has been generated using Nanopore MinION sequencing with an N50 of $2.98 \mathrm{Mb}$, yielding nine full chromosome-level sequences. This study adds to the current genomic resources available for the genus Alternaria and will provide opportunities for further analyses to unravel the mechanisms underlying pathogenicity of this important necrotrophic pathogen.
\end{abstract}

Alternaria brassicae is an important pathogen causing blight disease (also referred to as black spot) on most of the cultivated Brassica spp., particularly the oleiferous Brassica spp. (Conn and Tewari 1990; Nowicki et al. 2012). The genus Alternaria consists of more than 250 species that include both nonpathogenic and pathogenic species causing diseases on agronomically important cereal, vegetable, oilseed, and fruit crops and many ornamental species. A. brassicae represents one of the six monotypic lineages within the Alternaria genus (Woudenberg et al. 2013). The Alternaria spp. reportedly infecting the Brassicaceae family include $A$. brassicae, $A$. brassicicola, $A$. raphani, and, recently, $A$. alternata (Dunbar et al. 2017). It is hypothesized that primary infections are usually caused by the more invasive $A$. brassicae, while secondary infections are caused by $A$. brassicicola and $A$. alternata. In the Indian subcontinent, yield losses due to $A$. brassicae in the oilseed brassica crops range from 10 to $70 \%$ (Kolte et al. 1987). The losses are particularly high in the hilly regions and foothills of the mountain ranges where the climate is more conducive (cool and foggy) for infection and growth of $A$. brassicae. The high incidence of $A$. brassicae has practically restricted the cultivation of oilseed brassicas in these regions.

The genomes of many Alternaria spp., including that of $A$. brassicicola, have been published (Belmas et al. 2018; Dang et al. 2015; Hu et al. 2012; Nguyen et al. 2016; Wolters et al. 2018; Woudenberg et al. 2015). Many of the Alternaria spp. have been sequenced using short-read technologies and, thus, only draft genomes are available. Recently, a gapless genome assembly of $A$. solani has been made available (Wolters et al. 2018). The use of thirdgeneration sequencing technologies to obtain long reads offers a powerful approach to
Funding:

This work was supported by the grant from Department of Biotechnology, Ministry of Science and Technology, Government of India, under the project BT/IN/Indo-UK/CGAT/12/DP/2014-15.

\section{Keywords}

genomics, metabolomics, proteomics
Current address for Sivasubramanian Rajarammohan: National Agri-Food Biotechnology Institute, Mohali, India

${ }^{\dagger}$ Corresponding author: J. Kaur; jagreet@south.du.ac.in

The author(s) declare no conflict of interest.

Accepted for publication 26 March 2019. 
Table 1. Summary of genome assembly of Alternaria brassicae (J3) and its comparison with other Alternaria spp.

\begin{tabular}{|c|c|c|c|c|c|}
\hline Parameters & A. brassicae & A. solani ${ }^{a}$ & A. brassicicolab & A. alternata ${ }^{c}$ & A. alternata ${ }^{\mathrm{d}}$ \\
\hline Isolate name & J3 & altNL03003 & abra43 & $\mathrm{RL}-8442-2$ & SRC1IrK2f \\
\hline $\begin{array}{l}\text { Sequencing technology } \\
\text { Number of contigs }\end{array}$ & $\begin{array}{r}\text { Nanopore } \\
17\end{array}$ & $\begin{array}{c}\text { PacBio SMRT } \\
10\end{array}$ & $\begin{array}{c}\text { PacBio SMRT } \\
29\end{array}$ & $\begin{array}{c}\text { PacBio SMRT } \\
27\end{array}$ & $\begin{array}{r}\text { Illumina } \\
79\end{array}$ \\
\hline Number of contigs (>10,000 bp) & 17 & 10 & 29 & 25 & 55 \\
\hline Largest contig & $7,015,266$ & $6,940,169$ & $3,303,516$ & $3,968,015$ & $2,531,235$ \\
\hline Total length & $34,141,106$ & $32,779,142$ & $31,036,461$ & $33,499,641$ & $32,990,834$ \\
\hline N50 & $2,988,132$ & $2,866,555$ & $2,101,997$ & $2,828,041$ & $1,097,884$ \\
\hline GC (\%) & 50.7 & 51.32 & 50.85 & 50.95 & 51.4 \\
\hline
\end{tabular}

a Wolters et al. 2018.

b Belmas et al. 2018.

c Nguyen et al. 2016.

d Zeiner et al. 2016.

e PacBio SMRT = Pacific Biosciences single-molecule real-time.

produce high-quality genome assemblies at affordable costs. Long reads are currently generated from Pacific Biosciences single-molecule real-time sequencing technology and Oxford Nanopore sequencing technology. A contiguous assembly is important because genes encoding pathogenicity factors are often present in rapidly evolving repeat-rich regions of the genome, which are difficult to assemble (Thomma et al. 2016). Additionally, it has been shown that contiguous genome assemblies could lead to accurate prediction of complete gene models and secondary metabolite clusters, which are also important for pathogenicity (Dallery et al. 2017).

We report the genome sequencing and assembly of $A$. brassicae isolate $\mathrm{J} 3$ that was collected from $B$. juncea var. Varuna growing in our experimental field station at Jaunti, New Delhi, India (Rajarammohan et al. 2017). Isolate J3 was purified by single-spore isolation, maintained on a synthetic medium, and periodically passaged through $B$. juncea var. Varuna to maintain its virulence. We confirmed the identity of the species through analysis of its characteristic spore morphology, and sequences of the internal transcribed spacer (ITS) region and other conserved genes (GAPDH and RBP2). The sequences of the ITS region showed complete similarity to the sequences already reported for $A$. brassicae (Woudenberg et al. 2013), thus confirming the identity of the isolate.

High-molecular-weight genomic DNA was extracted from mycelia of 15-day-old in vitro grown cultures of $A$. brassicae. High-molecular-weight genomic DNA $(2 \mu \mathrm{g})$ was used for Nanopore library preparation using the LSK-108 ONT ligation protocol. The libraries were run on R9.4 SpotON MinION flowcells for $24 \mathrm{~h}$ with live base-calling enabled for all of the runs. The MinION runs produced 348,590 reads, amounting to $1.62 \mathrm{~GB}$ of data and approximately $46 \times$ coverage of the genome. The genome was assembled de novo using the Canu assembler, version 1.6 (Koren et al. 2017). Nanopolish was used to compute an improved consensus sequence using the signal-level raw data (Loman et al. 2015). The inherent error rates of long-read sequencing technologies are relatively higher than those of the short-read technologies. Although several improvements have been made in both the sequencing chemistry and base-calling software, the presence of errors in the final assembly and their effect on gene or protein prediction is the subject of ongoing debate and research (Koren et al. 2019; Watson and Warr 2019). Because no reference sequence is available for $A$. brassicae, we could not estimate the actual error rates in the assembly. However, we determined the sequence similarity of highly conserved genes (RBP2 and GAPDH) and regions (large subunit, small subunit, and ITS) of $A$. brassicae in our assembly, which was then compared with that available in the $\mathrm{NCBI}$ database and found them to be completely similar.

The $A$. brassicae genome assembly consisted of 17 contigs totaling $34.14 \mathrm{Mb}$ of sequences. The contigs ranged in length from $66.7 \mathrm{~kb}$ to $7.01 \mathrm{Mb}$. The $\mathrm{N} 50$ of the genome assembly was $2.98 \mathrm{Mb}$ (Table 1). The genome size of $A$. brassicae was found to be comparable with the genome size of other Alternaria spp. reported to date. Of the 17 contigs, 1 represented the mitochondrial genome. The circular nature of the contig representing the mitochondria could not be validated and, hence, we cannot affirm the completeness of the mitochondrial genome. Its size of approximately $189 \mathrm{~kb}$ showed that the mitochondrial genome of $A$. brassicae was significantly larger than those reported for other Alternaria spp. (Liao et al. 2017). A comparison of mitochondrial genomes of $A$. brassicae and A. alternata 
revealed high levels of similarity; however, the expansion in the size in $A$. brassicae was due to extensive duplications.

The presence of telomeric repeats at the ends of a contig has been considered to represent a complete chromosome (Seidl et al. 2015). We identified nine contigs with telomeric repeats on both ends. One other contig larger than $1 \mathrm{Mb}$ contained telomeric repeats on only one of the ends. Therefore, the current assembly contains nine complete chromosomes and one chromosome with telomeric repeats missing at one of the ends. Apart from these, there were six contigs, of which one of them was approximately $1 \mathrm{Mb}$ in size. These contigs may represent dispensable chromosomes and need to be characterized further.

We report a nearly complete, highly contiguous genome assembly for the necrotrophic pathogen $A$. brassicae using Nanopore sequencing, where nine chromosomes could be assembled telomere to telomere. This is one of the most complete genome assemblies within the Alternaria genus and adds to the list of phytopathogenic fungi with near-complete genomes. The reported genome assembly would enable both intraspecies and interspecies comparative genomic studies and their implications in pathogenesis.

The genome assembly and associated raw data have been deposited as NCBI BioProject PRJNA526360.

\section{Literature Cited}

Belmas, E., Briand, M., Kwasiborski, A., Colou, J., N'Guyen, G., lacomi, B., Grappin, P., Campion, C., Simoneau, P., Barret, M., and Guillemette, T. 2018. Genome sequence of the necrotrophic plant pathogen Alternaria brassicicola Abra43. Genome Announce. 6:e01559-17.

Conn, K. L., and Tewari, J. P. 1990. Survey of Alternaria black spot and Sclerotinia stem rot in central Alberta in 1989. Can. Plant Dis. Surv. 70:66-67.

Dallery, J. F., Lapalu, N., Zampounis, A., Pigné, S., Luyten, I., Amselem, J., Wittenberg, A. H. J., Zhou, S., de Queiroz, M. V., Robin, G. P., Auger, A., Hainaut, M., Henrissat, B., Kim, K. T., Lee, Y. H., Lespinet, O., Schwartz, D. C., Thon, M. R., and O'Connell, R. J. 2017. Gapless genome assembly of Colletotrichum higginsianum reveals chromosome structure and association of transposable elements with secondary metabolite gene clusters. BMC Genomics 18:667.

Dang, H. X., Pryor, B., Peever, T., and Lawrence, C. B. 2015. The Alternaria genomes database: A comprehensive resource for a fungal genus comprised of saprophytes, plant pathogens, and allergenic species. BMC Genomics 16:239.

Dunbar, M., Adhikari, A., Kontz, B., Varenhorst, A., Nleya, T., Byamukama, E., and Mathew, F. 2017. First report of Alternaria black spot caused by Alternaria alternata on Brassica carinata in South Dakota. Plant Dis. 101:1951.

Hu, J., Chen, C., Peever, T., Dang, H., Lawrence, C., and Mitchell, T. 2012. Genomic characterization of the conditionally dispensable chromosome in Alternaria arborescens provides evidence for horizontal gene transfer. BMC Genomics 13: 171.

Kolte, S. J., Awasthi, R. P., and Viswanath. 1987. Assessment of yield losses due to Alternaria blight in rapeseed and mustard. Indian Phytopathol. 40:209-211.

Koren, S., Phillippy, A. M., Simpson, J. T., Loman, N. J., and Loose, M. 2019. Reply to 'Errors in long-read assemblies can critically affect protein prediction'. Nat. Biotechnol. 37:127-128.

Koren, S., Walenz, B. P., Berlin, K., Miller, J. R., Bergman, N. H., and Phillippy, A. M. 2017. Canu: Scalable and accurate long-read assembly via adaptive $k$-mer weighting and repeat separation. Genome Res. 27:722-736.

Liao, M., Chen, C., and Li, Q. 2017. The complete mitochondrial genome of Alternaria alternata (Hypocreales: Nectriaceae). Mitochondrial DNA Part B 2:587-588.
Loman, N. J., Quick, J., and Simpson, J. T. 2015. A complete bacterial genome assembled de novo using only nanopore sequencing data. Nat. Methods 12: 733-735.

Nguyen, H. D., Lewis, C. T., Lévesque, C. A., and Gräfenhan, T. 2016. Draft genome sequence of Alternaria alternata ATCC 34957. Genome Announce. 4:e01554-15.

Nowicki, M., Nowakowska, M., Niezgoda, A., and Kozik, E. 2012. Alternaria black spot of crucifers: symptoms, importance of disease, and perspectives of resistance breeding. Veg. Crops Res. Bull. 76:5-19.

Rajarammohan, S., Kumar, A., Gupta, V., Pental, D., Pradhan, A. K., and Kaur, J. 2017. Genetic Architecture of resistance to Alternaria brassicae in Arabidopsis thaliana: QTL mapping reveals two major resistance-conferring loci. Front. Plant Sci. 8:260

Seidl, M. F., Faino, L., Shi-Kunne, X., van den Berg, G. C., Bolton, M. D., and Thomma, B. P. 2015. The genome of the saprophytic fungus Verticillium tricorpus reveals a complex effector repertoire resembling that of its pathogenic relatives. Mol. Plant-Microbe Interact. 28:362-373.

Thomma, B. P. H. J., Seidl, M. F., Shi-Kunne, X., Cook, D. E., Bolton, M. D., van Kan, J. A. L., and Faino, L. 2016. Mind the gap; seven reasons to close fragmented genome assemblies. Fungal Genet. Biol. 90:24-30.

Watson, M., and Warr, A. 2019. Errors in long-read assemblies can critically affect protein prediction. Nat. Biotechnol. 37:124-126.

Wolters, P. J., Faino, L., van den Bosch, T. B. M., Evenhuis, B., Visser, R. G. F., Seidl, M. F., and Vleeshouwers, V. G. A. A. 2018. Gapless genome assembly of the potato and tomato early blight pathogen Alternaria solani. Mol. Plant-Microbe Interact. 31:692-694.

Woudenberg, J. H., Groenewald, J. Z., Binder, M., and Crous, P. W. 2013. Alternaria redefined. Stud. Mycol. 75:171-212.

Woudenberg, J. H., Seidl, M. F., Groenewald, J. Z., de Vries, M., Stielow, J. B., Thomma, B. P., and Crous, P. W. 2015. Alternaria section Alternaria: Species, formae speciales or pathotypes? Stud. Mycol. 82:1-21.

Zeiner, C. A., Purvine, S. O., Zink, E. M., Paša-Tolić, L., Chaput, D. L., Haridas, S., Wu, S., LaButti, K., Grigoriev, I. V., Henrissat, B., Santelli, C. M., and Hansel, C. M. 2016. Comparative analysis of secretome profiles of manganese(II)-oxidizing ascomycete fungi. PLoS One 11:e0157844. 